\title{
Between Comments and Repeat Visit: Capturing Repeat Visitors with a Hybrid Approach
}

\author{
Jina Kim ${ }^{a, *}$, Yeonju Jang, ${ }^{a, *}$ Kunwoo Bae ${ }^{a}$, Soyoung $\mathrm{Oh}^{\mathrm{a}}$, Nam Jeong Jeong ${ }^{\mathrm{a}}$, \\ Eunil Park ${ }^{\mathrm{a}, * *}$, Jinyoung Han ${ }^{\mathrm{a}}$, Angel P. del Pobil ${ }^{\mathrm{a}, \mathrm{b}}$ \\ ${ }^{a}$ Sungkyunkwan University, Seoul, Korea \\ ${ }^{b}$ University Jaume-I, Castellon, Spain
}

\begin{abstract}
Purpose - Understanding customers' revisiting behavior is highlighted in the field of service industry and the emergence of online communities has enabled customers to express their prior experience. Thus, this study investigates customers' reviews on an online hotel reservation platform, and explores their postbehaviors from their reviews.
\end{abstract}

Design/methodology/approach - We employ two different approaches and compare the accuracy of predicting customers' post-behaviors: (i) using several machine learning classifiers based on sentimental dimensions of customers' reviews, and (ii) conducting the experiment consisted of two subsections. In the experiment, the first subsection is designed for participants to predict whether customers who wrote reviews would visit the hotel again (referred to as Prediction), while the second subsection examines whether participants want to visit one of the particular hotels when they read other customers' reviews (dubbed as Decision).

Findings - The accuracy of the machine learning approaches $(73.11 \%)$ is higher than that of the experimental approach (Prediction: 58.96\%, and Decision: $64.79 \%)$. The key reasons of users' predictions and decisions are identified through qualitative analyses.

\footnotetext{
*Equally contributed first authors

** Corresponding author, 312 International Hall, Sungkyunkwan University, 25-2 Sungkyunkwan-ro, Jongno-gu, Seoul 03063, Korea

Email address: eunilpark@skku.edu (Eunil Park)
}

Preprint submitted to Data Technologies and Applications

October 19, 2021 
Originality/value - The findings reveal that using machine learning approaches show the higher accuracy of predicting customers' repeat visits only based on employed sentimental features. With the novel approach of integrating customers' decision processes and machine learning classifiers, we provide valuable insights for researchers and providers of hospitality services.

Keywords: Online comments, repeat visit, machine learning, online reviews, user experience

\section{Introduction}

Considering online and mobile environments which easily provide a set of diverse information to potential customers, service providers make significant efforts on the interaction with the customers in both online and offline environments. Moreover, because customers can also express their previous experience by writing their reviews or attaching photographs on online communities, several scholars have investigated effects of the customers' expressions on other fustomers' decisions from a variety of perspectives, such as helpfulness (Otterbacher, 2009: Hu and Chen, 2016), usability, or user experience (Hedegaard and

10 Simonsen, 2013). Also, it has been reported that customers' evaluations in online and mobile environments including their reviews and ratings can be one of the projections in building their experience and satisfaction (Xiang et al., 2015. Zhao et al., 2019).

Since customers' re-purchasing or re-visiting behavior is one of the most important topics in the hospitality industry, many scholars have explored key determinants of their re-purchasing and re-visiting behavior (Um et al., 2006 Wu et al. 2018; Han et al., 2009, Petrick et al., 2001). Among identified determinants, customers' satisfaction is generally validated as one of the most influential antecedents of their repeated visits (Han et al., 2009) through the pnhancements of their loyalty (Kozak et al., 2005, Rust and Zahorik, 1993, Kim et al. 2015).

Thus, the current study unveils customers' repeat visits on hospitality ser- 
vices with the online reviews. We conduct the interview sessions with ten users to explore key factors that affects on their decisions in selecting one of the hotels in online hotel platform. After the sessions, customers' reviews and ratings were found to be the notable drives for decision making and the key information sources that are not provided by service providers. Moreover, based on the findings of several prior studies which indicated that customers' reviews are one of the important projections on their previous experience (Vermeulen and Seegers, 30 2009), the current study attempts to address the following research question (RQ):

- RQ: Can we capture customers' repeat visits using their reviews written after the first-visit?

In order to address this question, two approaches are employed. First, machine learning approaches with five well-known classifiers with the sentimental feature data extracted from customers' reviews were applied. Second, an Internet survey on 200 participants who have experience in using hotel reservation platforms was conducted with two subsections, Prediction and Decision. In the Prediction section, each participant was instructed to make a guess on whether 40 a customer who wrote a specific review on one of the hotels would visit the hotel again. In the Decision section, each participant was asked to make his/her own decision to reserve and use a hotel if he/she has to visit. After the survey, open-ended questionnaire items were presented to investigate and explore key reasons and standards of the selection procedures. Then, both quantitative and 45 qualitative analyses were conducted.

Based on the lessons learned from this study, several practical and academic implications are suggested from the viewpoints of both service providers and customers. The remainder of the current study is organized as follows. After describing the prior studies of customers' repeat visit in hospitality services and online reviews in literature review section, the interview sessions are presented to deeply explore experience on hotel online booking platforms. Then, the results of two approaches are examined. Based on the results and findings of 
the current study, both implications and concluding remarks are discussed.

\section{Related Work} terminants of their repeat visit.

\subsection{Reviews Written on Online Services}

With the introduction of online environments, online markets for each industry have also significantly grown. Considering the findings of previous studies in

\subsection{Repeat Visit in the Hospitality Industry}

In the increasingly competitive hospitality industry, providers of hospitality services attempt to strengthen their competitiveness by encouraging repeat visitors (Chi, 2012, Alegre and Cladera, 2009). With this trend, investigating both motivations and hindrances of customers' repeat visits is one of the important topics both for academic and industry researchers.

Among the determinants of repeat visits, customers' perceived satisfaction and quality formed by their experience are known as key determinants Alegre and Cladera, 2009). Moreover, both customers satisfaction and perceived service quality play notable roles that allow the customer to have a repeat visit

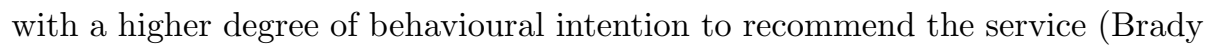
and Robertson, 2001). Similarly, Yoon and Uysal (2005) showed that two motivations, 'push' (customers' desires for relaxation, health, and social interaction), and 'pull' (tour destinations' attractiveness) effects on their behavioural intention to have a repeat visit.

Because there are numerous factors that affect customers' repeat visit and perceived satisfaction, a huge number of prior studies have examined such motivations with various approaches (Alegre and Cladera, 2009, Yoon and Uysal, 2005 Caruana, 2002 Kozak et al. 2005). Thus, based on the findings of prior studies on the roles of perceived satisfaction in the hospitality industry, this study assumes customers' satisfaction and their revealed experience as key dethe hospitality industry, over 70 percents of individual travel-related reservations 
and more than 30 percents of group travel-related reservations are examined in online environments (Schuckert et al. 2015). Because of the interactivity and influential effects on online environments and communications in our society, tourists easily share their experience, opinions, and information through their 85 individual websites, blogs, or social networking services (Leung et al., 2013 , Schuckert et al., 2015; Koh et al., 2010, Vermeulen and Seegers, 2009; Zhang et al. 2010).

In case of potential tourists, they can meet useful information of specific tour destinations which was generated by previous tourists who already visited the destinations ( $\mathrm{Li}$ and Hitt, 2008). As useful information sources, hotel reservation platforms that include a large number of reviews and ratings generated by customers can provide useful information for potential customers. This means that reviews and ratings of customers can be one of the notable outcomes and expressions of their prior experience. Thus, investigating and analyzing the reviews and ratings of customers can capture the customer experience, and consequently improve the overall quality of hospitality services.

\section{Exploratory Interview: Exploring Key Determinants in Hotel Se- lection}

We conducted exploratory interviews to investigate the effects of reviews and other features when deciding hotels. Considering the sample sizes of previous studies (Lian and Lin, 2008, Mitzner et al., 2010), we interviewed 10 participants who employed online hotel reservation platforms. All participants in the interview sessions were recruited from one of the private universities in South Korea. We recruited participants in their 20 s to 40 s, based on the findings on demographic traits of active users for the platforms (Kim et al., 2017). A brief information of the participants in the sessions is summarized in Table I] The participants' average age was 27.4, from 22 to 36 . Following the guidelines and instructions presented by Drever (1995), a semi-structured interview was conducted. Each session took about 15 minutes to complete. All interview items 
TABLE I. Participants' information in the interview sessions.

\begin{tabular}{lcrr}
\hline ID & Gender & Age & Frequency of Use in times/year \\
\hline P1 & M & 25 & 2 \\
P2 & F & 36 & $2-3$ \\
P3 & F & 23 & 1 \\
P4 & F & 27 & 5 \\
P5 & M & 31 & 2 \\
P6 & M & 22 & 2 \\
P7 & F & 28 & 2 \\
P8 & M & 30 & $1-2$ \\
P9 & F & 26 & $1-2$ \\
P10 & M & 26 & 1 \\
\hline
\end{tabular}

We extracted several user-oriented factors in the interview sessions. As presented in Figure 1. quality ratings, brands, price, images, amenities, locations, and other customers' evaluations including their reviews and ratings were identified. All participants responded to consider 'price' when they select a hotel. Both the location of hotels and other customers' reviews were also considered as notable factors in the selection $(90 \%)$.

The result indicates that customers preferred convenient locations which are close to major transportation points or key tourist attractions. Other customers' 125 reviews were also considered as one the main factors in selecting hotels. One of the participants mentioned as follows: 


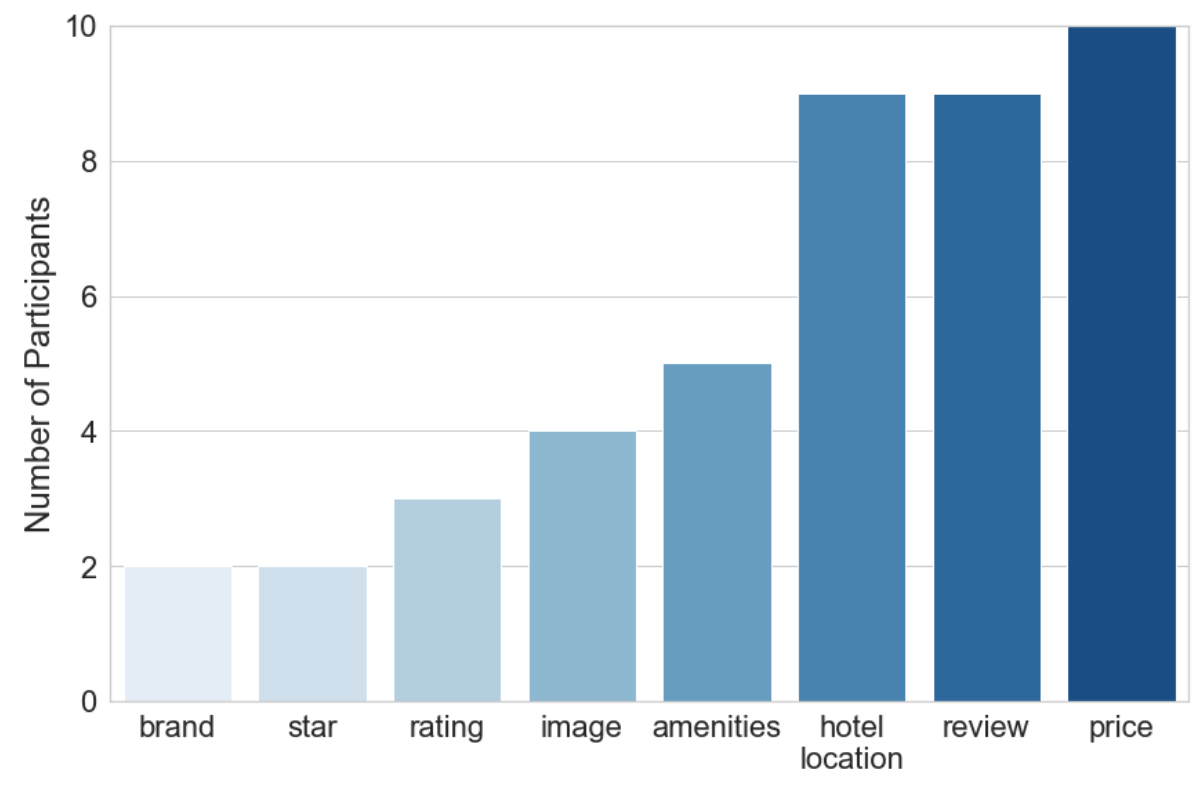

Figure 1. Results of the interview sessions.

- P2: "I tend to watch a lot of reviews. For instance, I check reviews on whether hotels provide a crib or a customer got bedbugs or fleas."

The majority of the participants indicated that they took a subjective view on other customers' reviews. Several participants tended to read more negative reviews than relatively positive review when they select the hotels, while some respondents only focused on positive reviews and ratings:

- P10: "I don't really read the reviews saying too positively about the hotels. I pay more attention to the negative ones."

- P5: "Because there are too many reviews, I just focus on the positive reviews and ratings."

Thus, in order to reveal how customers' reviews are associated with their repeat visits, we employed two approaches, (i) machine learning and (ii) survey, to predict whether customers have repeat visits or not. In case of the survey approaches, we conducted two sections, Prediction and Decision, for investigat- 
ing customers' selection procedures in two different situations, which will be detailed later.

\section{Study 1: Machine Learning Approaches}

\subsection{Data Collection}

145

We collected the 100,644 customers' data from one of the popular online hotel reservation platforms. The platform, which includes a huge number of hotels in over 200 countries and provides its services with about 90 languages, has been highly acknowledged as an authenticated and top worldwide hotel accommodation services (Banerjee and Bonfield, 2019). The data represents the customers' usage information of reserving hotels in the South Korean metropolitan area in 2012. After visiting the hotels, customers wrote reviews on the hotels in the platform. Later, they had a repeat visit between 2013 and 2016. Thus, we could identify whether customers again reserved the same hotel where they visited before; the customers who did not visit the same hotel reserved other hotels which were located within a 3000-meter radius of their previous visited hotel. Then, we pre-processed the collected data by removing reviews which are organized by commercial information. Also, we only considered the reviews written in English. Figure 2 presents our data pre-processing process.

\begin{tabular}{|c|c|c|}
\hline Users & \multicolumn{2}{|c|}{$\begin{array}{l}\text { All users visited Seoul in 2012, and } \\
\text { revisited Seoul between } 2013 \text { and } 2016\end{array}$} \\
\hline Reviews & \multicolumn{2}{|c|}{100,644} \\
\hline Employed Reviews & \multicolumn{2}{|c|}{75,422} \\
\hline Data Categorization & $\begin{array}{l}42,111 \\
\text { First Visit }\end{array}$ & $\begin{array}{c}\text { 33,311 } \\
\text { Repeat Visit }\end{array}$ \\
\hline
\end{tabular}

Figure 2. Summary of the collected reviews. 


\subsection{Features}

160 sessions: sentimental dimensions of reviews and ratings. To extract sentiment features from the reviews, we employed two text analysis tools, Linguistic Inquiry and Word Count (LIWC) and Valence Aware Dictionary and sEntiment Reasoner (VADER).

LIWC is a software tool which analyzes text in linguistic dimensions and psychological constructs. Although it is a commercial product, it has been commonly used to analyze user-generated contents in various hospitality services Amatulli et al. 2019, Ma et al., 2018, Hwang et al., 2020). Among 90 variables from LIWC output, we adopted summary variables including analytical thinking, emotional tone, clout, and authenticity, which were newly updated in the version of LIWC 2015 and a few other variables often employed in prior studies such as Word Count and affect (Hwang et al., 2020; Amatulli et al. 2019). The feature of affect represents one of the psychological processes indicating both positive and negative emotion. We then conducted extra trees feature selection method to identify influential variables in predicting repeat visits, resulting in three variables (i.e., analytic, tone, and affect).

The current study also employed VADER, an open-source sentiment analysis tool, which provides the results of sentiment polarity (i.e., positive and negative) of given texts (Gilbert and Hutto, 2014). We used both positive and negative scores from VADER, and ratings as input features. Thus, we employed two sets

of input features as follows:

- LIWC: three features (analytic, tone, and affect), and ratings

- VADER: two features (pos and neg), and ratings

Moreover, in order to present the appropriate degrees and distributions of sentimental features extracted from software tools, we used the reviews which have the top 25-percent of word counts. 


\subsection{Classification Models for Customer Repeat Visits}

We used five machine learning classifiers, which were employed in text-based classification tasks (Suchetha et al., 2019, Hwang et al. 2020): Logistic Regres-

190

fiers, to predict whether customers had a repeat visit on the hotel based on their reviews. We conducted grid search procedures to optimize hyperparameters of each classifier by repeating five-time five-fold cross validation steps. With regard to Logistic Regression, the liblinear solver with L2 penalty was used and in the model were extracted and provided by the results of the interview sessions. The information of hotel service providers, reviews and ratings presented by other customers in online hotel reservation platform were identified as key factors in selecting the hotels in the platform. In order to reflect customers' 215 the regularization coefficient ' $\mathrm{C}$ ' was set to 0.01 . We used the Gini index for Decision Tree, while both the max depth and max features of the tree was set to 3 . The number of neighbors was set to 13 for K-Neighbors classifier. Lastly, in the case of XGBoost classifier, the learning rate of 0.05 with 5 maximal depth was used. In addition, the minimum sum of weights and regularization parameter, gamma, were set to 3 and 0.1 respectively.

As shown in Table[I] we evaluated the machine learning classifiers using four different metrics: accuracy, precision, recall, and F1-score. Logistic Regression showed the highest accuracy (LIWC: 73.11\%). XGBoost (LIWC + VADER: $73.09 \%$ ) and Decision Tree (LIWC: $72.77 \%$ ) showed the following accuracy performances. In addition, Logistic Regression and Gaussian Naive Bayes showed the highest scores for precision and recall with VADER features $70.50 \%$ and $75.92 \%$ respectively.

\subsection{Implications}

We used machine learning approaches to predict whether customers who wrote specific reviews had a repeat visit on the hotel. The employed features various perspectives toward the reviews presented in the interview sessions, we extracted sentimental dimensions of reviews using two software tools, LIWC and 
TABLE II. Evaluation metrics of machine learning classifiers on true (repeat visit) class.

\begin{tabular}{cccccc}
\hline Model & Feature & Accuracy & Precision & Recall & F1-score \\
\hline \multirow{3}{*}{ Logistic Regression } & LIWC & $\mathbf{7 3 . 1 1}$ & 65.07 & 61.65 & 63.30 \\
& VADER & 72.80 & $\mathbf{7 0 . 5 0}$ & 47.60 & 56.83 \\
& LIWC+VADER & 73.08 & 65.02 & 61.61 & 63.26 \\
\hline \multirow{2}{*}{ Decision Tree } & LIWC & 72.77 & 61.76 & 73.13 & 66.55 \\
& VADER & 72.74 & 61.99 & 72.24 & 66.20 \\
& LIWC+VADER & 71.30 & 60.13 & 70.87 & 64.79 \\
\hline \multirow{3}{*}{ K-Neighbors } & LIWC & 69.77 & 60.32 & 57.40 & 58.81 \\
& VADER & 71.96 & 62.13 & 65.26 & 63.66 \\
& LIWC+VADER & 69.76 & 60.29 & 57.45 & 58.82 \\
\hline \multirow{3}{*}{ Gaussian Naive Bayes } & LIWC & 72.11 & 60.56 & 74.14 & 66.66 \\
& VADER & 72.41 & 60.65 & $\mathbf{7 5 . 9 2}$ & $\mathbf{6 7 . 4 3}$ \\
& LIWC+VADER & 70.94 & 59.12 & 73.82 & 65.65 \\
\hline \multirow{2}{*}{ XGBoost } & LIWC & 73.08 & 62.61 & 70.56 & 66.33 \\
& VADER & 72.85 & 62.17 & 71.22 & 66.31 \\
& LIWC+VADER & 73.09 & 62.72 & 70.15 & 66.17 \\
\hline
\end{tabular}

VADER. As the results of five machine learning classifiers, Logistic Regression employing a set of LIWC feature showed the highest accuracy of predicting the participants' repeat visit, $73.11 \%$. Consistent with the results of the interview sessions, customers' reviews are identified as the most significant factor, which represent their previous experience.

The current study reveals that sentimental features revealed in customers' reviews can be considered as key determinants of their repeat visits. Based on the results of the interview sessions, other customers' reviews and ratings are the most important factors in selecting one of the specific hotels in online hotel reservation platform. However, it is difficult to evaluate the performance and accuracy presented by these approaches, because we could not obtain ground truth information on the prediction of customers' repeat visit with their reviews and ratings. Thus, the current study conducts a survey on the prediction of customers' repeat visit with users who have experience in reserving and using a hotel room through the platform, in order to reflect potential subjective 
judgements of the users in repeat visits.

\section{Study 2: User Experiments on Repeat Visits}

A within-subjects experiment with two conditions was conducted to investi-

235

As the first section (Section 1: Prediction), participants were directly asked to predict the first-time visitor's repeat visit on the hotel, based on one of the customers' reviews on the hotel. In the second section (Section 2: Decision), participants were instructed to decide their own visitations on one of the specific

\subsection{Material Construction}

We designed hotel reservation websites, in order to provide interactive and realistic experiment environments. The process of producing survey materials was (1) sampling reviews, (2) capturing images of hotel detail page, and (3) combining reviews and images.

In this study, we aim to compare machine learning approach to human decision in utilizing reviews to predict repeat visits. Therefore, we constructed materials according to two significant standards for clear comparison: 1) The samples of reviews should be representative of data employed in the machine learning. 2) Other features besides reviews should be carefully controlled. We excluded those reviews which revealed hotel names and purpose of the visit among the reviews used in the machine learning for the second standard. Then, we randomly selected 120 reviews that showed the similar patterns with the employed features of LIWC to verify the first standard. The half of the reviews 60 non-repeat visitors (50\%). Then, considering the second standard, 24 images including the descriptions of hotel services and reviews were created.

The quality rating of selected hotels in the images were identically distributed from three to five stars, because the majority of the participants in 

presented.

\subsubsection{Prediction: Customers' Repeat Visit}

In Section 1, the following instructions were provided:

285

You will read a set of reviews on the hotels and make a guess whether a customer who wrote the review visited the hotel again. 
Based on the presented information in the website, all participants were the components and information on the website. Participants were required to explicitly explain the reasons about why they considered chosen components 
in each section. Lastly, a questionnaire item on the comparison between two sections was presented: 'If there is a significant difference between Sections 1 and 2, please explicitly explain the reasons'.

\subsection{Results}

\subsubsection{Accuracy}

The accuracy results of machine learning and survey approaches are summarized in Figure 3. In addition, Table III presents the summary of descriptive analysis on the accuracy with several socio-demographic categorizations.

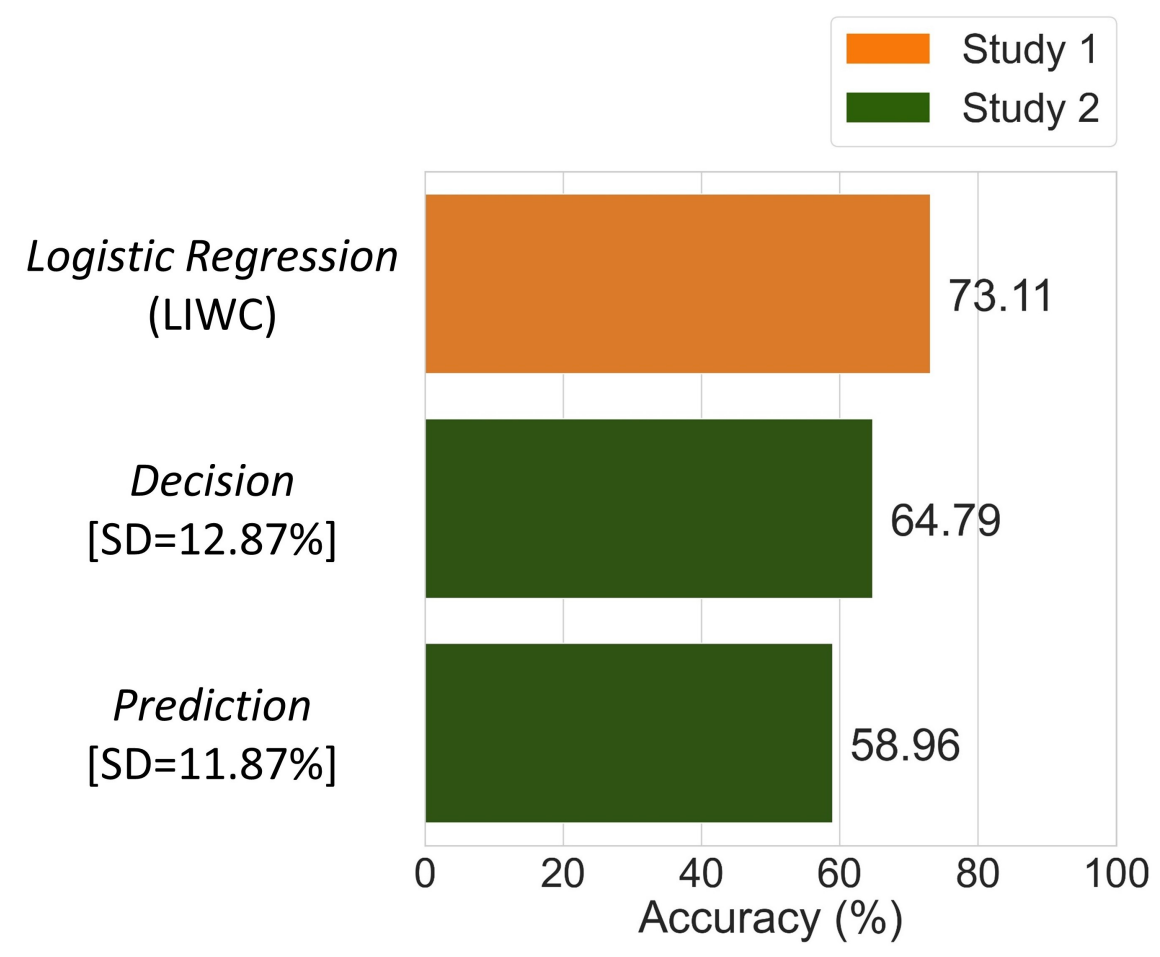

Figure 3. Results of machine learning and survey approaches.

An independent $t$-test was conducted to identify significant accuracy differences between Prediction and Decision. Participants reported significantly higher accuracy in Decision ( $M=64.79 \%, S D=12.87 \%$ ) than in Prediction $330 \quad(M=58.95 \%, S D=11.87 \%), F(1,398)=22.193, p<0.001$. 
TABLE III. Descriptive information on the accuracy

\begin{tabular}{llrr}
\hline \multirow{2}{*}{ Gender } & & Prediction & Decision \\
\hline \multirow{4}{*}{ Age } & Female & $59.17 \%(12.10 \%)$ & $67.33 \%(11.22 \%)$ \\
& Uale & $58.75 \%(11.69 \%)$ & $62.25 \%(13.94 \%)$ \\
\hline & $\mathbf{3 0 - 3 9}$ & $58.87 \%(10.94 \%)$ & $65.06 \%(12.10 \%)$ \\
& $\mathbf{4 0 - 4 9}$ & $58.33 \%(12.61 \%)$ & $66.49 \%(12.67 \%)$ \\
& Above 49 & $59.42 \%(10.45 \%)$ & $63.40 \%(11.98 \%)$ \\
\hline
\end{tabular}

Note. Mean (standard deviation)

\subsubsection{Information Sources}

We analyzed information sources that participants considered in the survey. At the end of the survey, participants were asked to respond a multiple-choice question for each section with sample images which indicate 9 notable information sources on the hotels (Figure 4a). Nine sources present the following information: 1) hotel name, 2) hotel quality (grade), 3) hotel address, 4) hotel images, 5) hotel amenities, 6) nearby attractions, 7) hotel map, 8) ratings, and 9) reviews.

The total number of information sources in both sections was computed (Figure 5a). The number of information sources per participant varied from one to nine. Participants considered more information sources when deciding their own visit intention, Decision (857), than predicting reviewers' repeat visit, Prediction (599).

We divided 9 sources into 2 groups based on the providers of sources (Figure 5b. Seven sources, 1) to 7), were introduced by hotel service providers (Source Group A) while the remaining 2 sources, 8) and 9), were offered by customers (Source Group B). The information introduced by service providers may have other purposes for potential customers (Kasper and Vela, 2011).

Participants in both sections reported that they highly considered the Source Group B (Figure 4b). For both sections, customers' ratings (8) and reviews (9) 


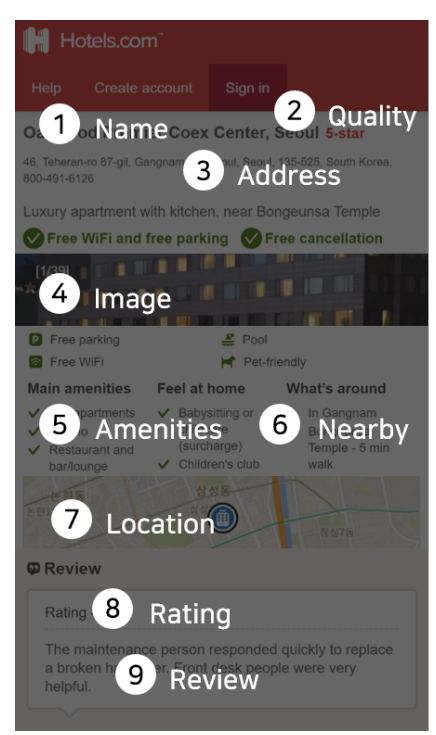

(a) An example of hotel reservation platform with the categorization of information sources

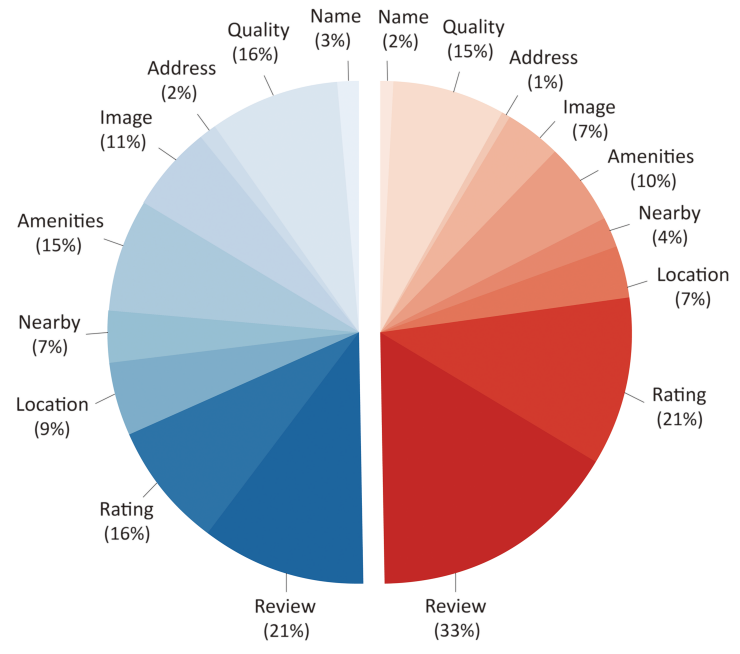

(b) The categorization of the responses (Blue: Prediction, Red: Decision)

Figure 4. Summary of information sources in Study 2.

were considered as two major factors among the 9 information sources.

Although 31 participants (among 200 participants) responded that they considered identical information sources in both sections, the ratios and distributions of the information sources are different for Section 1 (Prediction) and 2 (Decision). In detail, Figure $5 \mathrm{~b}$ indicates that the ratios of the Source Group B were more considered in Section 1 (Prediction), while the Source Group A were more used in Section 2 (Decision).

\section{Discussions}

To understand how customers' repeat visits are associated with the reviews with ratings, we conducted two studies. The results showed that the accuracy of machine learning classifiers (Logistic Regression: 73.11\%) was higher than that by human in the survey experiment. Also, the accuracy of the Section 1 (Decision: $64.79 \%$ ) was higher than that of the Section 2 (Prediction: 58.96\%). 


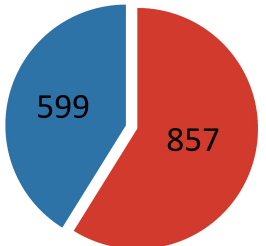

(a) The number of total responses (Blue: Prediction, Red: Decision)

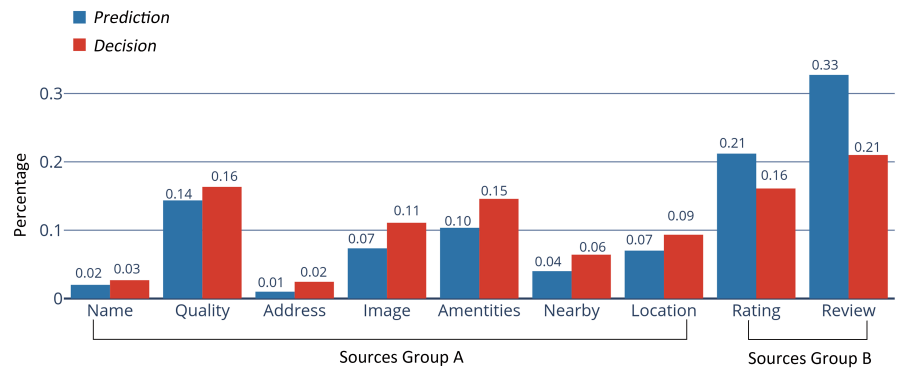

(b) Responses to information sources

Figure 5. Comparison between Prediction and Decision subsections in Study 2.

\subsection{Prediction versus Decision}

We further examined the results of the Prediction and Decision sections. We found that participants in the Decision considered more information than those in the Prediction. Information sources provided by the customers (i.e., Source Group B) were regarded as more significant than the ones provided by the hotel service providers (i.e., Source Group A) in both sections. Although the goals of both sections were different, several participants answered the same decision making processes for both sections. More than half of the participants $(55 \%)$ indicated that there is no significant difference between Prediction and Decision. Some participants reported as follows:

- P28, P45: "It was no different task. I did them with the same processes."

One of the potential reasons for this tendency can be explained by the theory of emotional contagion and projection. Generally, emotional contagion is defined as " a tendency to automatically mimic and synchronize expressions, vocalizations, postures and movements with those of another person and consequently, to converge emotionally" (Hatfield et al. 1993). Related to the current study, the participants can apply their own standards in deciding the hotels when they 
predict other customers' repeat visit. For instance, several participants showed the following expressions:

- P31: "If other customers can stay there, I can also stay!"

- P48: "I will be more willing to stay a specific hotel if I think other customers have repeat visit on the hotel."

- P114: "If I think that they did not stay there, usually I would not stay either."

- P30: "Like myself, if I stay at a hotel and things don't work, or outdate..bugs...tend to make me never want to go back. I expect these people are similar."

However, as presented in the results of accuracy and information sources, some participants (45\%) revealed the difference between Prediction and Decision processes. Representative responses were presented as follows:

- P158: "In the section of 'Decision', I thought of what I cared about. In the section of 'Prediction', I thought about what they cared about."

- P194: "Other customers' reviews are as per their needs, and the decision of reserving the hotel is as per our needs and comfort."

There can be several underlying reasons that the participants feel such differences. The concepts of (i) individual differences, (ii) information bias, (iii) involvement levels, and (iv) the number of visitation can be considered as the potential explanations.

\subsubsection{Individual Differences}

Some participants can consider their individual differences and characteristics between other customers who wrote reviews and themselves, which can result in difference between the participants' prediction of other customers' behavior and their own decision. That is, because each participant had his/her 
own standards in selecting one of the hotels, it may be more difficult to understand other customers' standards in the Prediction. However, since the participant need not to understand other customers' standards in the Decision, the participant tended to observe and refer to more information provided by the platform.

- P81: "It was different because I don't know what the other customers prefer in terms of pictures, amenities, and so on. I could only go by their reviews. But when deciding whether I would stay, I know exactly what I want."

- P173: "My own decision making took into consideration of all factors that influenced how "I" would view the property. I cannot see into the minds of other customers to know what is important to them so I focused only on their reviews."

\subsubsection{Involvement Levels}

The involvement level of the participants can be one of the explanations which the participants consider more information sources in the Decision, compared to the Prediction. In users' decision making procedures, the involvement level is one of the widely-employed determinants in mainly affecting their decisions (Sirakaya and Woodside, 2005). In the section of Decision, the participants tended to explore and obtain more information than other tasks.

- P85: "I felt that, since I was going to stay at the hotel, I took the information beyond just the reviews more seriously."

In addition, the purchasing behavior are required to include a set of procedures such as recognition, information search, evaluation of alternatives, or considerations of hindrances. That is, the participants in the Decision tended to find the presented information of products or services similar to the procedures of purchasing and using the products or services.

- P177: "The discrepancy between the star rating and the user rating, a 5 star hotel with a 1 star review would make me not want to book there." 


\subsubsection{Information Bias}

There can be the effects of information bias on the participants' selections. Some participants did not refer to other customers' reviews in the Decision, because they considered that the majority of reviews are negatively biased. Moreover, the concepts of information bias on customers' reviews can be presented with the opinion polarization (e.g., extremely satisfied and dissatisfied users) (Anderson, 1998).

- P134: "Reviews...I consider, but then remember that some people will always complain no matter what and that many who leave reviews tend to be unhappy whereas happy customers do not feel the need to vent online."

\subsubsection{First-time and Repeat Visits}

The differences between the first-time and repeat visits can provide notable explanations with the considerations of information sources for the Decision and Prediction. Participants in the Prediction took part in predicting other customers' repeat visit, while in the Decision, they were required to respond each question for their first visit. Several prior studies have investigated that there can be remarkable differences of users' motivations, loyalty levels, and pvaluations between first-time and repeat visits (Lim et al., 2016, Chi, 2012, Li et al. 2008).

Considering the notable differences on the characteristics of the first-time and repeat visits, different information sources were employed for each section. Generally, information sources can be categorized into internal (e.g., personal experience, knowledge) and external (e.g., market-oriented factors) sources (Jacobsen and Munar, 2012; Lee and Cranage, 2010). The repeat visit is highly affected by the internal information sources, while the first-time visit is more influenced by the external information sources. Because customers' reviews are formed by their previous experience, the Source Group B can be more considered in the Prediction than the Source Group A.

- P102: "The only reason it is different is because the rating and review 
encompassed everything in their experience, so they are not necessary to predict if the reviewer would stay there again. All that matters is how they perceived their stay. When deciding to book a reservation, I don't have any experience yet, so I have to rely on multiple different factors to form an opinion on whether I want to stay there or not."

- P171: "There really was very little difference. You use the same methods to book a hotel first time, but then you would rely on your own personal experience at said hotel for a decision to repeat a visit or not."

\subsection{Machine Learning versus Survey Approaches}

We can find several similar factors which can be considered to predict customers' repeat visit through their reviews between machine learning and human in user experiments. We employed tone, affect, and analytic features when using machine learning. Similarly, the participants in the survey study showed that they mainly considered both positive and negative emotional statements of reviews. Moreover, the participants responded that there are notable effects of positive emotional statements presented in the reviews on the positive selection (53.5\%, in the Prediction and the Decision). In addition, there were significant effects of negative emotional statements in the reviews on the negative selections in both sections $(71 \%)$.

- P33: "I made my decisions based on the positive and negative reviews."

- P197: "I pretty much went on good reviews vs. bad reviews."

\subsubsection{Differences: Source Credibility}

The concept of source credibility can be one of the possible explanations on the results of machine learning approaches which showed the greater accuracy than those by human through the survey. It is defined as "a source's perceived ability (expertise) or motivation (trustworthiness) to provide accurate and truthful information" ( $\mathrm{Hu}$ and Shyam Sundar, 2010). Because the participants' predictions and decisions include the considerations of source credibility 
and their own evaluations (Nan, 2009, Tormala and Petty, 2004), it may have negative impacts on the results.

the survey approaches. With these limitations, future studies can extend the findings of the current study in improving machine learning models which can more accurately predict customers' behavior including their repeat visits. 


\section{Acknowledgements}

520 Korea, under the ICAN (ICT Challenge and Advanced Network of HRD) program (IITP-2020-0-01816) supervised by the IITP (Institute of Information \& Communications Technology Planning \& Evaluation). This work was supported by the National Research Foundation of Korea (NRF) grant funded by the Korea 525

\section{References}

Alegre, J., Cladera, M., 2009. Analysing the effect of satisfaction and previous visits on tourist intentions to return. European Journal of Marketing 43, $670-685$.

530 Amatulli, C., De Angelis, M., Stoppani, A., 2019. Analyzing online reviews in hospitality: Data-driven opportunities for predicting the sharing of negative emotional content. Current Issues in Tourism 22, 1904-1917.

Anderson, E.W., 1998. Customer satisfaction and word of mouth. Journal of service research $1,5-17$.

535 Banerjee, S., Bonfield, S., 2019. How online reviews in a year predict online sales in the next on expedia. com + agoda. com + hotels. com? a panel study of hotels, in: 2019 5th International Conference on Information Management (ICIM), IEEE. pp. 336-340.

Brady, M.K., Robertson, C.J., 2001. Searching for a consensus on the antecedent role of service quality and satisfaction: an exploratory cross-national study. Journal of Business research 51, 53-60.

Caruana, A., 2002. Service loyalty: The effects of service quality and the mediating role of customer satisfaction. European journal of marketing 36, 811-828. 
Chi, C.G.q., 2012. An examination of destination loyalty: Differences between first-time and repeat visitors. Journal of Hospitality \& Tourism Research 36, $3-24$.

Corbin, J., Strauss, A., 2008. Strategies for qualitative data analysis, in: Basics of Qualitative Research (3rd ed.): Techniques and Procedures for Developing Grounded Theory. Sage Publications Inc, pp. 85-105.

Drever, E., 1995. Using Semi-Structured Interviews in Small-Scale Research. A Teacher's Guide. ERIC.

Gilbert, C., Hutto, E., 2014. Vader: A parsimonious rule-based model for sentiment analysis of social media text, in: Eighth International Conference on Weblogs and Social Media (ICWSM-14). Ann Arbor, MI., p. 82.

Han, H., Back, K.J., Barrett, B., 2009. Influencing factors on restaurant customers' revisit intention: The roles of emotions and switching barriers. International Journal of Hospitality Management 28, 563-572.

Hatfield, E., Cacioppo, J.T., Rapson, R.L., 1993. Emotional contagion. Current directions in psychological science 2, 96-100.

Hedegaard, S., Simonsen, J.G., 2013. Extracting usability and user experience information from online user reviews, in: Proceedings of the SIGCHI Conference on Human Factors in Computing Systems, ACM. pp. 2089-2098.

Hu, Y., Shyam Sundar, S., 2010. Effects of online health sources on credibility and behavioral intentions. Communication research 37, 105-132.

Hu, Y.H., Chen, K., 2016. Predicting hotel review helpfulness: The impact of review visibility, and interaction between hotel stars and review ratings. International Journal of Information Management 36, 929-944.

Hwang, S., Kim, J., Park, E., Kwon, S.J., 2020. Who will be your next customer: A machine learning approach to customer return visits in airline services. Journal of Business Research 121, 121-126. 
Jacobsen, J.K.S., Munar, A.M., 2012. Tourist information search and destination choice in a digital age. Tourism management perspectives 1, 39-47.

Kasper, W., Vela, M., 2011. Sentiment analysis for hotel reviews, in: Computational linguistics-applications conference, pp. 45-52.

575 Kim, M., Vogt, C.A., Knutson, B.J., 2015. Relationships among customer satisfaction, delight, and loyalty in the hospitality industry. Journal of Hospitality \& Tourism Research 39, 170-197.

Kim, S.Y., Kim, J.U., Park, S.C., 2017. The effects of perceived value, website trust and hotel trust on online hotel booking intention. Sustainability 9, 2262.

${ }_{580}$ Koh, N.S., Hu, N., Clemons, E.K., 2010. Do online reviews reflect a product's true perceived quality? an investigation of online movie reviews across cultures. Electronic Commerce Research and Applications 9, 374-385.

Kothari, C.R., 2004. Research methodology: Methods and techniques. New Age International.

Kozak, M., Bigné, E., Andreu, L., 2005. Satisfaction and destination loyalty: A comparison between non-repeat and repeat tourists. Journal of Quality Assurance in Hospitality \& Tourism 5, 43-59.

Lee, C.H., Cranage, D.A., 2010. Customer uncertainty dimensions and online information search in the context of hotel booking channel. Journal of Hospitality Marketing \& Management 19, 397-420.

Leung, D., Law, R., Van Hoof, H., Buhalis, D., 2013. Social media in tourism and hospitality: A literature review. Journal of travel \& tourism marketing $30,3-22$.

Li, X., Hitt, L.M., 2008. Self-selection and information role of online product reviews. Information Systems Research 19, 456-474. 
Li, X.R., Cheng, C.K., Kim, H., Petrick, J.F., 2008. A systematic comparison of first-time and repeat visitors via a two-phase online survey. Tourism Management 29, 278-293.

Lian, J.W., Lin, T.M., 2008. Effects of consumer characteristics on their acceptance of online shopping: Comparisons among different product types. Computers in human behavior 24, 48-65.

Lim, Y.J., Kim, H.K., Lee, T.J., 2016. Visitor motivational factors and level of satisfaction in wellness tourism: Comparison between first-time visitors and repeat visitors. Asia pacific journal of tourism research 21, 137-156.

Ma, E., Cheng, M., Hsiao, A., 2018. Sentiment analysis-a review and agenda for future research in hospitality contexts. International Journal of Contemporary Hospitality Management 30, 3287-3308.

Mitzner, T.L., Boron, J.B., Fausset, C.B., Adams, A.E., Charness, N., Czaja, S.J., Dijkstra, K., Fisk, A.D., Rogers, W.A., Sharit, J., 2010. Older adults talk technology: Technology usage and attitudes. Computers in human behavior $26,1710-1721$.

Nan, X., 2009. The influence of source credibility on attitude certainty: Exploring the moderating effects of timing of source identification and individual need for cognition. Psychology \& Marketing 26, 321-332.

Otterbacher, J., 2009. 'helpfulness' in online communities: a measure of message quality, in: Proceedings of the SIGCHI conference on human factors in computing systems, ACM. pp. 955-964.

Park, E., Kang, J., Choi, D., Han, J., 2020. Understanding customers' hotel revisiting behaviour: a sentiment analysis of online feedback reviews. Current Issues in Tourism 23, 605-611.

Petrick, J.F., Morais, D.D., Norman, W.C., 2001. An examination of the determinants of entertainment vacationers' intentions to revisit. Journal of Travel Research 40, 41-48. 
Polman, E., Vohs, K.D., 2016. Decision fatigue, choosing for others, and selfconstrual. Social Psychological and Personality Science 7, 471-478.

Rust, R.T., Zahorik, A.J., 1993. Customer satisfaction, customer retention, and market share. Journal of retailing 69, 193-215.

Schuckert, M., Liu, X., Law, R., 2015. Hospitality and tourism online reviews: Recent trends and future directions. Journal of Travel \& Tourism Marketing $32,608-621$.

Sirakaya, E., Woodside, A.G., 2005. Building and testing theories of decision making by travellers. Tourism management 26, 815-832.

Suchetha, N., Nikhil, A., Hrudya, P., 2019. Comparing the wrapper feature selection evaluators on twitter sentiment classification, in: 2019 International Conference on Computational Intelligence in Data Science (ICCIDS), IEEE. pp. 1-6.

Tormala, Z.L., Petty, R.E., 2004. Source credibility and attitude certainty: A metacognitive analysis of resistance to persuasion. Journal of Consumer Psychology 14, 427-442.

Um, S., Chon, K., Ro, Y., 2006. Antecedents of revisit intention. Annals of tourism research $33,1141-1158$.

Vermeulen, I.E., Seegers, D., 2009. Tried and tested: The impact of online hotel reviews on consumer consideration. Tourism management 30, 123-127.

Wu, H.C., Li, M.Y., Li, T., 2018. A study of experiential quality, experiential value, experiential satisfaction, theme park image, and revisit intention. Journal of Hospitality \& Tourism Research 42, 26-73.

Xiang, Z., Schwartz, Z., Gerdes Jr, J.H., Uysal, M., 2015. What can big data and text analytics tell us about hotel guest experience and satisfaction? International Journal of Hospitality Management 44, 120-130. 
${ }_{650}$ Yoon, Y., Uysal, M., 2005. An examination of the effects of motivation and satisfaction on destination loyalty: a structural model. Tourism management $26,45-56$.

Zhang, Z., Ye, Q., Law, R., Li, Y., 2010. The impact of e-word-of-mouth on the online popularity of restaurants: A comparison of consumer reviews and editor reviews. International Journal of Hospitality Management 29, 694-700.

Zhao, Y., Xu, X., Wang, M., 2019. Predicting overall customer satisfaction: Big data evidence from hotel online textual reviews. International Journal of Hospitality Management 76, 111-121. 